\title{
BRIEF: \\ 6 WAYS TO INCORPORATE SOCIAL CONTEXT AND TRUST IN INFODEMIC MANAGEMENT
}

Information epidemiology or infodemiology is the study of infodemics - defined by the World Health Organization as an overabundance of information, some accurate and some not, that occurs during a pandemic or other significant event that may impact public health. Infodemic management is the practice of infodemiology and may sit within the risk communication and community engagement (RCCE) pillar of a public health response. However, it is relevant to all aspects of preparedness and response, including the development and evaluation of interventions. Social scientists have much to contribute to infodemic management as, while it must be data and evidence driven, it must also be built on a thorough understanding of affected communities in order to develop participatory approaches, reinforce local capacity and support local solutions.

\section{INFODEMIOLOGY AND COMMUNITY ENGAGEMENT}

This brief explains how social science can inform infodemiology by making it more attuned to different social, political and cultural contexts and to the relationships between people and formal institutions. By providing an understanding of the contexts and cultures in which infodemics circulate, social scientists can help infodemic managers to work with people to make decisions based on the most accurate and appropriate information. This brief will first set out what needs to be done and then offer six ways to do it. The brief was developed by the Social Science in Humanitarian Action Platform (SSHAP) led by Santiago Ripoll, and by Jennifer Cole, Royal Holloway University of London, a WHO Infodemic Manager. The brief is the responsibility of the SSHAP.
Information should be reliable, factually correct and appropriate for the context. High-quality information can spread faster than disease, encouraging early adoption of protective behaviours and building trust in reliable sources.

Misinformation is false or inaccurate information, including rumours, shared in the belief that it is correct. Misinformation can fill a void where information is lacking, particularly when information from authorities and officials is slow to emerge, contradictory, confusing or inconsistent.

Disinformation is information that is intentionally false or misleading, and may be spread for political, economic or social gain to advance a particular agenda. 


\section{WHAT NEEDS TO BE DONE: SUPPORT PEOPLE TO MAKE DECISIONS BASED ON ACCESSIBLE, ACCURATE AND ACTIONABLE INFORMATION}

During a pandemic, people seek more information, from more sources than usual in order to make sense of their situation. Infodemiology is not only about guiding people to the best and most trustworthy information but also about understanding how they seek information, how they communicate with others, why they trust some pieces of information and not others, and how best to include them in formulating new and more appropriate information and messages. Through dialogue and inclusion, they will become partners in the co-creation of knowledge, the embedding of better understanding and the adoption of the most effective behaviours.

The political and social context in which information is circulating is at least as important as the quality of information. It may not matter how accurate information is if people are not prepared to believe it. If they do not believe it, they will not act on it. How people receive information depends on many factors: who they are (gender, ethnicity, class etc.), their health literacy, their lived experience (of the epidemic and of previous health service access and availability) how public health measures affect them and whether they feel prioritised or marginalised in the response. Messages must be appropriate to local contexts. Understanding underlying challenges and tensions will help to explain which messages are more likely to resonate.

Just providing accurate information is not enough to ensure people make the right decisions. Information must be delivered through trusted sources. Misinformation gains traction and multiplies when trust in government authorities and health providers is low. But trust is a process: it cannot be built overnight. Trust may be particularly low among groups who face discrimination and marginalisation, but mistrust is also found among majority groups, particularly in contexts where their relationship with government and official sources has been damaged previously by corruption or failure. Reaching communities will depend on establishing dialogue with people and channels they already trust. Tailoring approaches to and engagement with different groups to build acceptance is a critical part of infodemic management. If communities are not engaged, misinformation, confusion and mistrust can more easily undermine public health efforts.

Tackling misinformation may inadvertently strengthen the claims of those who spread it. Banning misinformation and the channels over which it is shared, or penalising people who create and/or circulate inaccurate information, can backfire by adding credence to claims of government or corporate censorship and repression of alternative views. This can push such discussions underground, making it more difficult to identify or to engage constructively. Infodemic managers must understand where misinformation originates, how it is spreading and why it resonates if it is to be successfully challenged. 
Building trust in public health authorities and epidemic response takes time and is an ongoing process. However, in the short term, mistrust can be mitigated by responding in contextually appropriate ways through meaningful community engagement:

1. Use social science to understand the socio-economic, political and historical context in which information is circulating. The worldviews of an affected community will impact how information is filtered. Local contexts and cultural framings can shape meanings and influence how information is received, interpreted and shared, and determine which voices are most trusted. Recognizing this will help to identify key influencers and platforms, common ground, potential allies and main challenges.

2. Adapt communications to respond to the concerns of different groups of people, using trusted sources and platforms. There are many affected communities, facing common challenges but each has unique needs. It is important to adapt messages to reflect the diversity of audiences - offline and online. Use language, rationales and justifications that appeal directly to target groups. Consider how different meanings could be attributed to particular phrases or images and what feelings these might evoke. Test all communications outputs (videos, posters, messages) with intended audiences. Focus on understanding the role of local news media, advertising platforms, artistic representations and community platforms as well as online media.

3. Establish dialogue and create feedback systems. People need to be able to express their views, opinions and concerns and freely ask questions that will be answered by people they trust. Appropriate suggestions should be incorporated into response and future plans to ensure people feel included and heard. Perceptions can change as events unfold: engagement needs to be ongoing to understand why and to identify barriers and enablers that influence capability to sustain, as well as to adopt, positive health behaviours. Behavioural change is more likely to be successful and sustainable if the community is directly involved in developing solutions from the earliest stages.

4. Include diverse groups and listen with an open mind - misinformation and rumours are influenced by people's life experiences and current situation. Information and communications should be empathetic rather than judgemental or patronising. Infodemic management needs to engage with multiple stakeholders, including media agencies and platforms through which messages will be disseminated. Consultations should include representatives of vulnerable and marginalised groups who understand the practical challenges faced by their communities, and the origins of concerns and barriers. Place them at the centre of efforts to engage communities and build relationships based on pre-established trust. 
5. Be transparent, consistent and open, particularly about uncertainty, controversy and mistakes. Be honest about what is being done in response to the epidemic. Be open about what is known and unknown and where there is uncertainty; prepare audiences for the likelihood that advice may change. Be clear about how decisions have been made and transparent about who played a role in decision-making (e.g. pharmaceutical companies, private outsourcing, the role of decentralised authorities, representatives of minorities, etc.). If new information suggests that mistakes have been made, be honest about this and explain what is being done to address them.

6. Offer compelling narratives that build a sense of capability and motivation to act. Develop accurate messages that explain the truth clearly rather than only dismissing misinformation and debunking myths. The same information reaches different audiences: understand who these audiences are and what their distinct, and potentially competing concerns may be. Recognise where concerns originate and identify what messages are more likely to generate positive emotional response and a sense of togetherness and solidarity. Social scientists can support infodemic managers to engage local communities, build trust and co-create solutions and messages that are more likely to lead to effective responses.

\section{OTHER RESOURCES}

- Tangcharoensathien, $V$., et al. 2020. Framework for managing the COVID-19 infodemic: methods and results of an online, crowdsourced WHO technical consultation. Journal of medical Internet research, 22(6), p.e19659.

- Butler, N \& Cole, J. 2020. Online Information, Mis-and Disinformation in the Context of COVID-19 (SSHAP) https://www.socialscienceinaction.org/resources/keyconsiderations-online-information-mis-disinformation-context-covid-19/

- COVID-19 Global Risk Communication and Community Engagement Strategy December 2020-May 2021. https://www.who.int/publications/i/item/covid-19-globalrisk-communication-and-community-engagement-strategy

\section{ACKNOWLEDGMENTS}

This paper is co-authored by Santiago Ripoll (Institute of Development Studies - IDS), Jennifer Cole (Royal Holloway University of London and a WHO Infodemic Manager), Olivia Tulloch (Anthrologica), Megan Schmidt-Sane (IDS) and Tabitha Hrynick (IDS). We would like to thank Jon Bugge, independent RCCE consultant and Christine Czerniak for expert input. 
If you have a direct request concerning the response to COVID-19, regarding a brief, tools, additional technical expertise or remote analysis, or should you like to be considered for the network of advisers, please contact the Social Science in Humanitarian Action Platform by emailing Annie Lowden (a.lowden@ids.ac.uk) or (oliviatulloch@anthrologica.com). Key Platform liaison points include: UNICEF (nnaqvi@unicef.org); IFRC (ombretta.baggio@ifrc.org); and GOARN Research Social Science Group (nina.gobat@phc.ox.ac.uk).
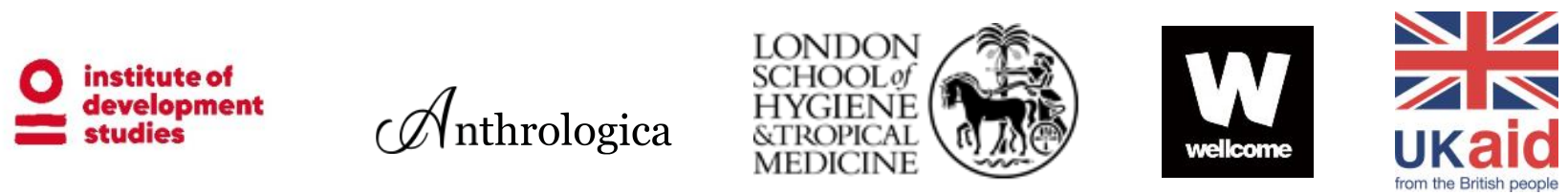

The Social Science in Humanitarian Action is a partnership between the Institute of Development Studies, Anthrologica and the London School of Hygiene and Tropical Medicine. Funding to support the Platform's response to COVID-19 has been provided by the Wellcome Trust and FCDO. The opinions expressed are those of the authors and do not necessarily reflect the views or policies of IDS, Anthrologica, LSHTM, Wellcome Trust or the UK government.

Suggested citation: Ripoll, S., Cole, J., Tulloch, O., Schmidt-Sane, M and Hrynick, T: '6 Ways to Incorporate Social Context and Trust in Infodemic Management', Brighton: Social Science in Humanitarian Action (SSHAP) DOI: 10.19088/SSHAP.2021.001

Published January 2021

(c) Institute of Development Studies 2021

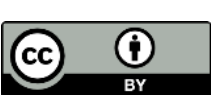
This is an Open Access paper distributed under the terms of the Creative Commons Attribution 4.0 International licence (CC BY), which permits unrestricted use, distribution, and reproduction in any medium, provided the original authors and source are credited and any modifications or adaptations are indicated. http://creativecommons.org/licenses/by/4.0/legalcode 